\title{
An osteoarthritis triple play
}

\author{
Friedrich C. Luft $^{1}$
}

Published online: 27 May 2016

(C) Springer-Verlag Berlin Heidelberg 2016

In American baseball, a triple play is the rare act of making three outs during the same continuous play. In this issue of $\mathrm{JMol} \mathrm{Med}$, we feature a triple play against the world's most common disease. Osteoarthritis (OA) is a degenerative joint disease and the commonest form of arthritis affecting about one third of adults over age 45 years. Since most of these persons seek medical treatment, osteoarthritis is a massive worldwide healthcare and financial burden. Articular cartilage loss, subchondral bone thickening, and osteophyte formation characterize the condition. A cartoon outlining the process is shown (Fig. 1). While new targets are emerging to treat osteo-arthritis [1], palliative treatments with non-steroidal anti-inflammatory drugs (NSAIDs) and corticosteroid injections remain the most common therapies. Eventually, hip and knee replacement become necessary in many patients. Furthermore, much back surgery is a sequel to OA. Not uncommonly, physicians prescribe opioids, such as oxycodone when NSAIDs fail. As a result, osteoarthritis is a major contributor to the entire pain industry.

$\mathrm{OA}$ is associated with many contributing factors including aging, obesity, trauma, genetics, and other accompanying causes; however, basic molecular mechanisms are not fully understood. Debate remains as to what is really the precipitating pathology, since everyone eventually has OA. Poulet and Staines have underscored the focus on different joint tissues affected by OA, including a focus on articular cartilage maintenance, targeting subchondral bone, inhibiting osteoclast activity, modifying osteoblast behavior, and adjusting angiogenesis [2].

Friedrich C. Luft

luft@charite.de

1 Experimental and Clinical Research Center, Charite Medical Faculty and the Max Delbrück Center for Molecular Medicine, Lindenbergerweg 80, Berlin 13125, Germany
Moon et al. recently reported in this journal that mice lacking pannexin 3 (Panx3) specifically in cartilage were markedly resistant to the development of OA following destabilization after medial meniscus surgery [3]. Their data indicated a specific catabolic role of Panx3 in articular cartilage maintenance and identified Panx 3 as a potential therapeutic target for OA. Their report was the first in vivo evidence for a role of Panx3 in disease. Since three additional investigations into OA are reported by this journal following the paper by Moon and colleagues, examining the subject again appears highly justified.

Ko and colleagues also concerned themselves with articular cartilage maintenance [4]. They point out that heat-shock proteins (HSPs), intracellular chaperones, orchestrate tissue homeostasis and are important in remodeling and repair. They studied the articular cartilage from OA patients undergoing knee surgery and found reduced HSP60 concentrations in cartilage and synovial fluid compared to specimens from non-OA patients. Furthermore, expression of the SRY-box 9 (SOX9) chondrogenic transcription factor was reduced. They next investigated chondrocyte cultures that were stimulated with interleukin-1 $\beta$ (IL-1 $\beta$ ). This exposure reduced HSP60, SOX9, collagen II, and aggrecan expression; however, the reductions were ameliorated when HSP60 was restored. To pursue the issue further, the investigators studied transgenic (Tg) HSP60 overexpression in cartilage of mice. These mice had articular cartilage that was $25 \%$ increased in thickness. An OA knee model involving collagenase injections showed that the TgHSP60 mice were resistant to this insult and osteophyte development was reduced. The investigators found that HSP60 signaling maintained SOX9 expression by attenuating SOX9 ubiquitinylation in affected joints. The findings were underscored by the observation that lentivirus-shuttled HSP60 into affected knees mitigated cartilage destruction and synovitis.

Similarly exciting is the recent report in $J$ Mol Med by Ji and colleagues [5]. These investigators focused on 
Fig. 1 Osteoarthritis features cartilage loss, subchondral bone thickening, osteophyte formation, and joint destruction. Illustration from http://medical-dictionary. thefreedictionary.com/ osteoarthritis with permission

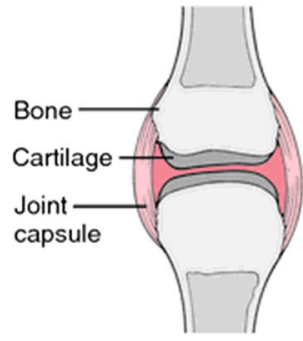

NORMAL

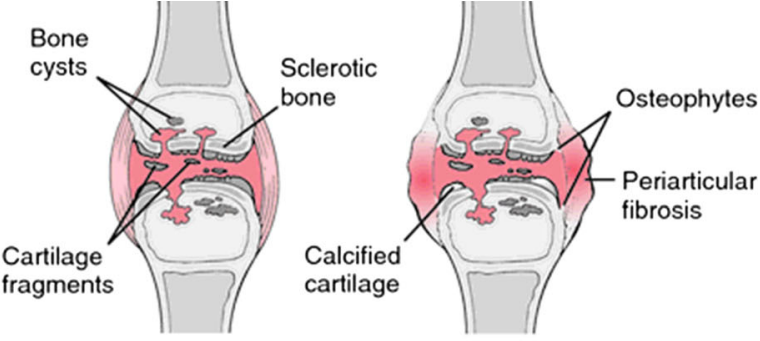

OSTEOARTHRITIS

Irregular joint space Fragmented cartilage Loss of cartilage Sclerotic bone Cystic change
OSTEOARTHRITIS - ADVANCED

Osteophytes

Periarticular fibrosis

Calcified cartilage mechanisms of cartilage destruction in OA. They pointed out that IL- $1 \beta$ is involved in the mechanistic progression of OA by stimulating matrix-degrading proteases, particularly the "disintegrin metalloproteinase with thrombospondin motifs5" (ADAMTS-5). Genetically modified mice in which the catalytic domain of ADAMTS-5 was deleted are resistant to cartilage destruction in an experimental model of osteoarthritis [6]. The investigators demonstrated that IL-1 $\beta$ regulates ADAMTS-5 expression by suppressing microRNA-30a. They showed that microRNA-30a expression was downregulated in OA patients and was inversely correlated with ADAMTS-5 expression. The IL- $1 \beta$ regulation involved the activator protein (AP-1) transcription factor c-Jun/c-Fos that bound to the microRNA-30a promoter. This pathway could represent a therapeutic target to inhibit the development and progression of OA.

In their review, Poulet and Staines emphasized that increased angiogenic activity leading to increased vascular invasion of the subchondral bone has been reported in the early stages of OA in both animal models and in human patients [2]. Indeed, Zhang et al. investigated just this problem as they report now in $\mathrm{J} \mathrm{Mol} \mathrm{Med} \mathrm{[7].} \mathrm{They} \mathrm{used} \mathrm{a} \mathrm{lentivirus-based}$ approach to investigate whether or not a vascular endothelial growth factor (VEGF) knockdown would be beneficial to chondrogenesis and thereby slow the progression of OA. They first profiled cytokine expression in human cartilage
Fig. 2 The cytokines IL-1 $\beta$ and TNF- $\alpha$ are two of many. Shown is data suggesting that via HSP60 and SOX9, OA can be influenced and that HSP60 introduction is protective. Next, IL-1b can induce a microRNA regulating a crucial matrix metalloproteinase contributing to OA. Finally, cytokine receptors influence angiogenesis and its inhibition influences the development of $\mathrm{OA}$

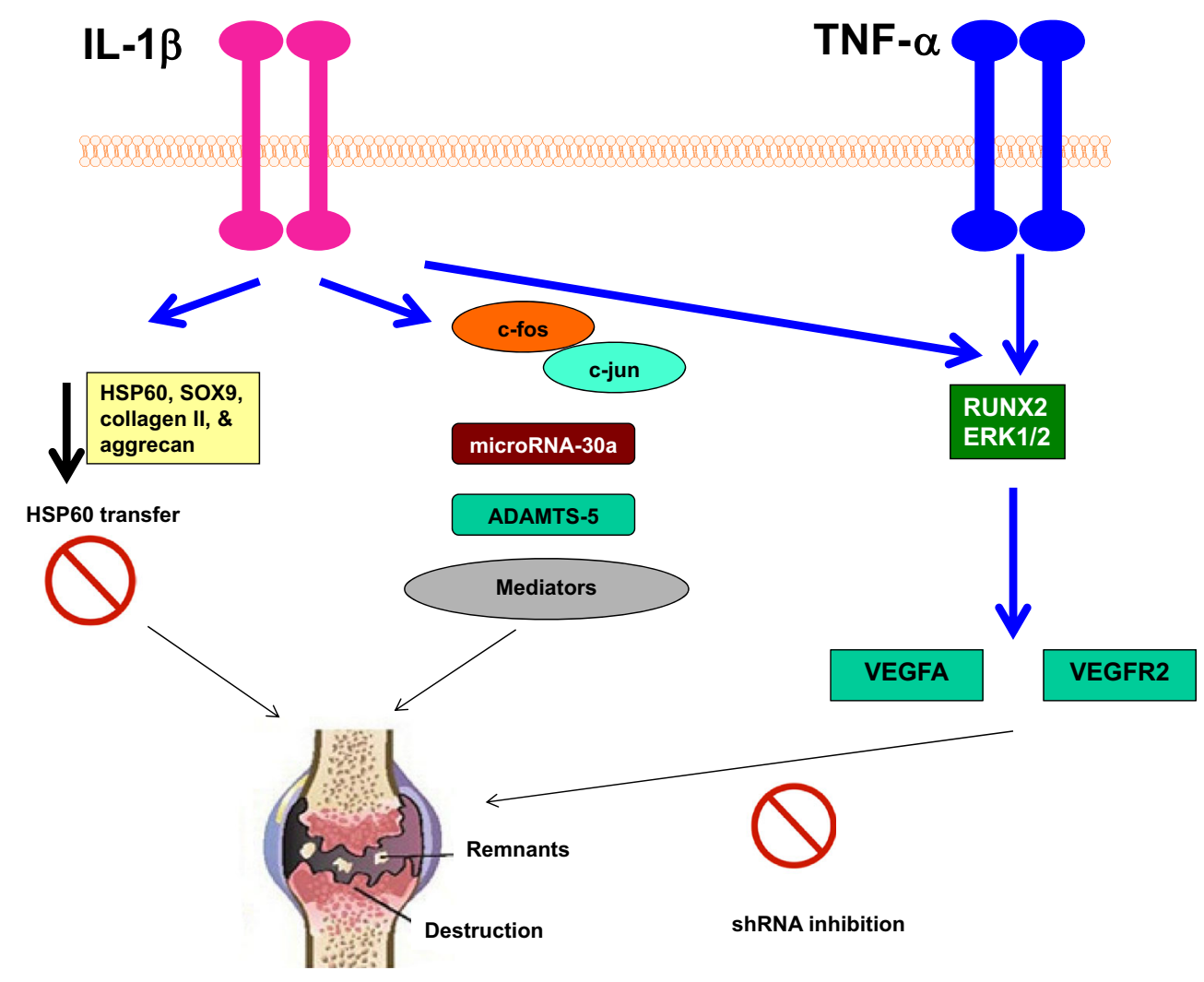


from OA patients. Twenty-one candidates were identified. Next chondrocytes were treated with tumor necrosis factor- $\alpha$ (TNF- $\alpha$ ) to induce hypertrophy. VEGF inhibition with shRNA protected the cells from hypertrophy and also enhanced chondrogenic differentiation. VEGF inhibition also suppressed TNF-a-induced extracellular-regulated kinase (ERK1/2) phosphorylation. Next, the investigators tested the findings in a rat model. They showed that VEGF inhibition delayed OA progression in rats given intra-articular injections of lentiviral-delivered VEGF shRNA. Thus, their approach may have therapeutic ramifications.

To do these three papers justice in a single illustration (Fig. 2) is a challenge. We observe that cytokine receptors (numerous candidates were found, while only two were investigated in these studies) are pivotal. We learn that chaperones (HSP60) are important; although we received no information on what is being chaperoned here, we discovered another microRNA regulating an important pathway, and we learned a bit more about angiogenesis. All of these findings are important in unraveling a major, clinically important puzzle.

Respectfully,

Friedrich C. Luft

\section{References}

1. Goldring MB, Berenbaum F (2015) Emerging targets in osteoarthritis therapy. Curr Opin Pharmacol 22:51-63

2. Poulet B, Staines KA (2016) New developments in osteoarthritis and cartilage biology. Curr Opin Pharmacol 28:813

3. Moon PM, Penuela S, Barr K, Khan S, Pin CL, Welch I, Attur M, Abramson SB, Laird DW, Beier F (2015) Deletion of Panx3 prevents the development of surgically induced osteoarthritis. J Mol Med 93: 845-856

4. Ko JY, Sun YC, Li WC, Wang FS. (2016) Chaperonin 60 regulation of SOX9 ubiquitination mitigates the development of knee osteoarthritis. J Mol Med. doi:10.1007/s00109-016-1422-3

5. Ji Q, Xu X, Zhang Q, Kang L, Xu Y, Zhang K, Li L, Liang Y, Hong T, Ye Q et al (2016) The IL-1beta/AP-1/miR-30a/ADAMTS-5 axis regulates cartilage matrix degradation in human osteoarthritis. J Mol Med. doi:10.1007/s00109-016-1418-Z

6. Glasson SS, Askew R, Sheppard B, Carito B, Blanchet T, Ma HL, Flannery CR, Peluso D, Kanki K, Yang Z et al (2005) Deletion of active ADAMTS5 prevents cartilage degradation in a murine model of osteoarthritis. Nature 434: 644-648

7. Zhang X, Crawford R, Xiao Y. (2016) Inhibition of vascular endothelial growth factor with shRNA in chondrocytes ameliorates osteoarthritis. J Mol Med. doi:10.1007/s00109-016-1425-0 